\title{
Enunciación
}

\section{Prácticas de literacidad en la clase de biología en secundaria}

\author{
Literacy practices in the middlle school biology class
}

\section{Diana Marcela Buitrago Díaz ${ }^{1}$, Erika Alejandra Naranjo², Nubia Johana Cifuentes Baquero³, Emilce Moreno Mosquera ${ }^{4}$}

Para citar este artículo: Buitrago, D.M.; Naranjo, E.A.; Cifuentes, N.J. y Moreno, E. (2016). Prácticas de literacidad en la clase de biología en secundaria. Enunciación, 21(2), 272-287.

Recibido: 30-abril-2016 / Aprobado: 7-septiembre-2016

\section{Resumen}

En este artículo se analizan las prácticas de lectura y escritura de la clase de biología, en el grado séptimo de dos instituciones. El objetivo de esta investigación fue conocer cómo son las prácticas de literacidad en esta asignatura, y qué las distingue en relación al campo disciplinar, de ahí que este estudio se ubique en el marco de estudio de la literacidad disciplinar. Se trata de observar y analizar las prácticas de lectura y escritura escolares articuladas al mundo social, a partir de determinadas formas de actuar, sentir, creer y pensar. Se realizaron diez observaciones y se entrevistaron a las profesoras del área. A partir de ello, se identificaron las prácticas y los eventos letrados más comunes, los cuales fueron interpretados a través de las siguientes categorías: literacidad disciplinar, evento letrado, comunidad de discurso y dominio del área por parte del estudiante. De otro lado, se encontró, entre las concepciones de las profesoras acerca de la importancia de la lectura y la escritura, que dichas prácticas se asocian al uso de la lectura como recurso de investigación; al uso de la escritura como medio de producción desde conceptos interiorizados y la enseñanza de la biología como una posibilidad de formar el pensamiento científico.

Palabras clave: lectura, escritura, conocimiento científico, enseñanza de la biología, escuela secundaria.

\begin{abstract}
This paper analyzes the reading and writing practices in the biology class of the seventh grade in two different institutions. The aim of this research is to know how literacy practices are on this subject, and what differences are between them in relation to the disciplinary field. Therefore, this study locates in the disciplinary literacy framework. In this article, the authors observe and analyze the school's reading and writing practices articulated in the social world beginning on certain ways of acting, feeling, believing and thinking. Ten observations were made and the teachers in the subject were interviewed. As result, the most common practices and literacy events were identified and interpreted by the following categories: disciplinary literacy, literacy
\end{abstract}

1 Pontificia Universidad Javeriana de Bogotá. Correo electrónico: dmbuitrago@javeriana.edu.co

2 Pontificia Universidad Javeriana de Bogotá. Correo electrónico: naranjo.e@javeriana.edu.co

3 Pontificia Universidad Javeriana de Bogotá. Correo electrónico: n.cifuentes@javeriana.edu.co

4 Docente e investigadora de la Facultad de Educación de la Pontificia Universidad Javeriana de Bogotá. Correo electrónico: moreno-e@javeriana.edu.co 
event, discourse community, and the subject domain by the student. On the other hand, other finding is among the conceptions of the teacher about the importance of reading and writing that such practices are associated with the use of reading as a research resource; the use of writing as a meaning of

\section{INTRODUCCIÓN}

La literacidad, entendida como práctica social que se desarrolla en cualquier espacio o momento de la vida, refleja las costumbres, tradiciones o rutinas que se adoptan debido al contexto situacional. También es asumida como aquello que forma parte del proceso de enseñanza y aprendizaje, que involucra la lectura y la escritura como prácticas indispensables para la apropiación del conocimiento, así como del lenguaje específico; en el caso de la biología, la terminología científica, cuya interiorización debe ser de manera gradual teniendo en cuenta la edad y el grado escolar. De acuerdo con Moje (2007), la literacidad disciplinar tiene como premisa que el conocimiento de las disciplinas es siempre discursivo y mediado por textos; en esa medida, conviene examinar las formas orales y escritas de producir ese conocimiento en cada disciplina. En cada contexto se establecen relaciones entre los sujetos implicados que llevan a compartir formas características de hablar, actuar, evaluar, interpretar y usar el lenguaje escrito (Barton y Hamilton, 2000).

En especial se retoma la literacidad disciplinar debido a que el trabajo en ciencias, como construcción social, se distancia del conocimiento producido por otras disciplinas y por el conocimiento cotidiano. En la enseñanza de las ciencias se establecen relaciones conceptuales a partir de fenómenos asociados a la naturaleza y a los seres vivos; se busca el sentido de estos fenómenos a través de la observación, la formulación de preguntas, la argumentación y la explicación, además, se reconoce una terminología. Se trata de involucrar a los estudiantes en la generación de conocimiento, para production from internalizing concepts and biology teaching as a possibility to way scientific thinking.

Keywords: reading, writing, scientific knowledge, biology teaching, middle school.

lo cual participan en prácticas epistémicas de la ciencia en las que proponen, justifican, evalúan y legitiman los contenidos en un determinado marco teórico disciplinar (Duschl y Osborne, 2002). De ahí la preocupación de diferentes investigadores en torno a las prácticas epistémicas de la ciencia y el papel de la lectura, la escritura, la oralidad y la argumentación en estas (Longino, 1990; Lemke, 1997; Jiménez y Sanmartí, 1997; Duschl, 2002; Márquez y Prat, 2005).

En el aula se abordan los hechos científicos con un carácter impersonal y funcionan como realidades aparentemente objetivas:

El conocimiento científico es aquel cuyas descripciones de fenómenos naturales, que la comunidad científica establece con un carácter impersonal y que opera como realidad por apoyarse en lo que se establece como "evidencia empírica" -a la que se le atribuye un carácter de objetividad-, es producido con una forma de aparente neutralidad, como independiente de los sujetos y de las condiciones sociales de producción y que, por tanto, se establece como verdad (Gilbert y Mulkay, 1984; Potter, 1996, como se citó en Candela, 2012, p.44).

En esa medida, en el lenguaje científico prevalecen los criterios racionales, la explicación y la argumentación. El abordaje de los fenómenos naturales implica verificarlos objetivamente, sin embargo, como lo plantea Candela (2012) la evidencia empírica no necesariamente es objetiva, puesto que:

Los sujetos interpretan la realidad a partir de las concepciones que tienen. En diversos estudios sobre la interacción discursiva en el aula se encuentra que "lo que se ve" para los docentes, frecuentemente no 
"se ve" para los alumnos ya que ellos interpretan la realidad de una manera diferente. Sin embargo, también se observa que en clases de ciencias es permanente la alusión a la "evidencia empírica" como mecanismo de legitimación de la validez de una versión (pp.46-47).

Tomando como punto de partida la riqueza de las prácticas de literacidad de la clase de biología, en este estudio se abordó una perspectiva etnográfica para dar cuenta de las prácticas de lectura y escritura que caracterizan esta disciplina, en el grado seleccionado. De ahí que lectura y escritura se entiendan como prácticas sociales construidas por medio de códigos y actividades que a su vez son moldeadas por los lectores y los escritores en la relación con los textos. Las prácticas de literacidad cobran forma por "las reglas sociales que regulan el uso y la distribución de los textos, y prescriben quiénes pueden producirlos y quiénes tienen acceso a ellos" (Barton y Hamilton, 2000, p.8). El posicionamiento en esta perspectiva y en el ámbito de la literacidad como tal, supone la comprensión de los siguientes referentes conceptuales: i) evento letrado; ii) práctica letrada; iii) la comunidad discursiva, y iv) las categorías planteadas por Hamilton (2000).

Los eventos letrados se entienden como aqueIlos episodios observables de lectura o escritura que se dan en escenarios de la vida o contextos sociales específicos. Barton y Hamilton (2000) explican que son actividades donde la literacidad cumple un papel fundamental, en la medida en que se emplean textos en diferentes tareas y se da lugar a conversaciones desde el contenido del texto trabajado. La práctica letrada se refiere a "la relación entre las actividades de lectura y escritura y las estructuras sociales, en las cuales se integran y toman forma" (Barton y Hamilton, 1998, p.6). Estas prácticas "involucran valores, actitudes, sentimientos y relaciones sociales que son procesos internos del individuo y que no siempre son observables" (Zavala, 2008, p.28). De otro lado, se encuentran las categorías planteadas por Barton y Hamilton (2000) asociadas a elementos visibles y no visibles de las prácticas de lectura y escritura. En la tabla 1 se presentan los elementos implicados en el análisis de prácticas y eventos de literacidad.

Tabla 1. Elementos básicos para analizar prácticas y eventos de literacidad

\begin{tabular}{|c|c|c|}
\hline & $\begin{array}{c}\text { Elementos visibles en los eventos } \\
\text { de literacidad }\end{array}$ & $\begin{array}{c}\text { Elementos constitutivos (no visibles) de las } \\
\text { prácticas de literacidad }\end{array}$ \\
\hline Participantes & $\begin{array}{l}\text { Las personas que se pueden ver interac- } \\
\text { tuando con los textos escritos. }\end{array}$ & $\begin{array}{l}\text { Los participantes ocultos -otras personas o grupos } \\
\text { de personas involucrados en las relaciones socia- } \\
\text { les de producción, interpretación, circulación y } \\
\text { regulación de los textos escritos-. }\end{array}$ \\
\hline Escenario/contexto & $\begin{array}{l}\text { Las circunstancias físicas inmediatas en } \\
\text { las cuales se lleva a cabo la interac- } \\
\text { ción. }\end{array}$ & $\begin{array}{l}\text { El dominio de la práctica dentro del cual el evento } \\
\text { se desarrolla y adquiere sentido y propósito social. }\end{array}$ \\
\hline Artefactos & $\begin{array}{l}\text { Las herramientas materiales y los acce- } \\
\text { sorios involucrados en la interacción } \\
\text { (incluidos los textos). }\end{array}$ & $\begin{array}{l}\text { Todos los otros recursos que se integran a la } \\
\text { práctica de la literacidad, incluidos los valores no } \\
\text { materiales, los entendimientos (sobreentendidos), } \\
\text { las formas de pensar y de sentir, las habilidades y } \\
\text { los conocimientos. }\end{array}$ \\
\hline Actividades & $\begin{array}{l}\text { Las acciones llevadas a cabo por los } \\
\text { participantes durante el evento de } \\
\text { literacidad. }\end{array}$ & $\begin{array}{l}\text { Rutinas y trayectorias estructuradas que facilitan o } \\
\text { regulan las acciones. Las reglas de adecuación y } \\
\text { elegibilidad -quién participa o no, quién puede o } \\
\text { no participar en actividades particulares-. }\end{array}$ \\
\hline
\end{tabular}

Fuente: Barton y Hamilton (2000, p.16). 
Se trata de las categorías asociadas a la descripción y análisis de elementos implicados en las prácticas de literacidad: participantes, escenario, artefactos, actividades, que posibilitan la comprensión de los modos en que los estudiantes acceden al conocimiento disciplinar y participan en prácticas de lectura y escritura. De igual modo, en la interacción entre los estudiantes y los profesores se promueve la generación de una comunidad discursiva, es decir, la asimilación y aculturación de las convenciones retóricas de los discursos en los cuales se involucra al estudiante; según Cassany (2008), esta comunidad es:

Un grupo humano que comparte unas prácticas comunicativas particulares, usando unos textos particulares, para conseguir unas finalidades específicas, entre autores y lectores que asumen roles predeterminados. A través de esas prácticas y de sus textos, los miembros de la comunidad llegan a compartir conocimientos específicos, unas habilidades cognitivas para procesarlos, unas actitudes y una determinada mirada sobre la realidad, que son exclusivas del grupo y que acaban constituyéndose como una marca de identidad frente a profesionales de otros ámbitos y frente a comunidades de otros ámbitos. Ser miembro de una comunidad implica conocer los mecanismos de producción, transmisión y recepción del conocimiento que manejan sus miembros; dominar los géneros discursivos con que se materializa la comunicación entre sí y la construcción de dicho conocimiento; poder (des)codificar los diferentes sistemas de representación del conocimiento empleados; asumir los roles, el estatus y la identidad que se adopta, además de haber adquirido los recursos lingüísticos específicos (terminología, fraseología, estructura del texto, etc.) (p.12).

Teniendo como marco lo anterior, la pregunta que guía esta investigación es: “¿Cómo son las prácticas de literacidad en la asignatura de biología en el grado séptimo en dos instituciones educativas?". Esto supone analizar el uso de la lectura y la escritura en los contextos seleccionados, específicamente, el Colegio Colombo Español, de Barranquilla, y Liceo María Inmaculada, de Bogotá. Así mismo, implica identificar los eventos letrados involucrados en la apropiación del lenguaje científico y cómo los textos contribuyen al aprendizaje del mismo. En esa medida, se busca analizar las prácticas de literacidad en la asignatura de biología en las instituciones mencionadas. A partir de lo cual se identifican prácticas comunes implicadas en la lectura y la escritura de los textos, así como, los eventos letrados extraídos a partir de la observación. El análisis también supone la reflexión sobre algunas implicaciones de la construcción de saberes en ciencias a partir de las observaciones y las entrevistas a las profesoras.

\section{METODOLOGÍA}

En concordancia con los objetivos de este estudio y con el marco de la literacidad, se adoptó un enfoque etnográfico, el cual posibilitó la observación y el registro de los eventos letrados y el acercamiento a las rutinas mediadas por los textos que dan sentido a las prácticas de literacidad. Para Goetz y LeCompte (1984) el objeto de la etnografía educativa es "aportar valiosos datos descriptivos de los contextos, actividades y creencias de los participantes en los escenarios educativos" (p.41). Esto supone que la observación de los eventos letrados conduce a una información mediada por prácticas sociales con participantes concretos.

Los instrumentos que se utilizaron para la recolección de información fueron los registros de observación, que permitieron dar cuenta de todo lo que sucedía en el aula, las transcripciones de la clase y las entrevistas a las docentes de las instituciones, a fin de conocer sus prácticas y concepciones en torno a la relevancia de la lectura y la escritura en su asignatura. Para la recolección de la información y la clasificación de los eventos letrados se tuvo en cuenta el registro empleado por Araceli de Tezanos (1998). Este registro de observación permite la identificación de los participantes y los aportes que cada uno de ellos hace al desarrollo de la clase. En adelante utilizaremos estas notaciones en la ejemplificación de los turnos. 
M - Maestro.

$\mathrm{A}^{\circ}$ - Alumno.

Aa -Alumna.

Algs. $\mathrm{A}^{\circ} \mathrm{s}-$ Algunos alumnos.

" "Registro textual.

O - Observador.

(..) Duda del hablante o no se entiende lo dicho.

A partir del trabajo de observación se tuvo en cuenta: i) la reflexión analítica de los datos, ii) la selección y reducción de los datos y iii) la organización y categorización de los datos. Según lo planteado por Hammersley y Atkinson (1994), "las necesidades del etnógrafo, por supuesto, a la hora de decidir qué códigos son relevantes para los temas del trabajo en cuestión y para el análisis preliminar que acompaña a la recogida de información, son prioritarias" (p. 217). Inicialmente se hizo una codificación de la información recopilada, de un lado las transcripciones de las clases observadas, de otro, las dos entrevistas realizadas con las profesoras. A partir de ello, se definieron las siguientes categorías para la parte de las observaciones: literacidad disciplinar, evento letrado, comunidad de discurso y dominio del área por parte del estudiante. En relación con las entrevistas, las categorías fueron: a) La utilización de la lectura como recurso de investigación; b) la utilización de la escritura como medio de producción desde conceptos interiorizados y c) la enseñanza de la biología como una posibilidad de formar el pensamiento científico. Este procedimiento deductivo en principio se realizó siguiendo a Krippendorf (1990), a partir de la identificación de los códigos se reconocieron categorías amplías de tipo analítico, de manera que puedan conectarse estas con los segmentos de datos textuales.

\section{ANÁLISIS DE LA INFORMACIÓN}

\section{El aula como foco de análisis}

Teniendo en cuenta lo observado en las clases de la asignatura de biología, se identificaron de modo deductivo cuatro categorías, guiadas inicialmente por los planteamientos teóricos de algunos autores y luego complementadas con el trabajo de campo realizado, estas son: literacidad disciplinar, evento letrado, comunidad de discurso y dominio de la asignatura por parte del estudiante.

\section{Literacidad disciplinar}

Como ya se señaló, la lectura y la escritura forman parte de las tareas cotidianas en diferentes disciplinas, y son esenciales para la formación. Precisamente esta categoría se asocia con las prácticas de lectura y escritura que son propias de la asignatura observada. Algunas de ellas son: explicaciones apoyadas en imágenes, lectura en voz alta, explicación a partir de lo leído en el libro de texto, desarrollo de actividades para abordar el tema (completar taller), el dictado.

Debido a la complejidad de la materia, una de las estrategias más relevante fue la utilización de las imágenes como herramientas de refuerzo para lograr que los estudiantes pudieran interiorizar los conceptos trabajados en el aula; por otro lado, está la necesidad de los estudiantes de dibujar para entender o ubicarse en el tema, por ejemplo:"la nutrición del ser humano" (figura 1), o "la respiración en los animales" (figura 2). En el ejemplo que se cita a continuación, los estudiantes se encuentran abordando estos temas y preguntan:

\section{Algs. $A^{\circ}$ s: ¿Cómo hacemos? ¿El dibujo primero y luego escribimos?}

En los ejemplos que se citan a continuación, se muestran segmentos de cómo los estudiantes registran las explicaciones sobre los temas "la nutrición del ser humano" en la figura 1 y "la respiración en los animales" en la figura 2, en los cuales se puede ver la imagen con las denominaciones de las partes y el texto explicativo.

La mayoría de los estudiantes toman apuntes y afianzan la explicación de la profesora con la elaboración de dibujos que evidencian lo trabajado 


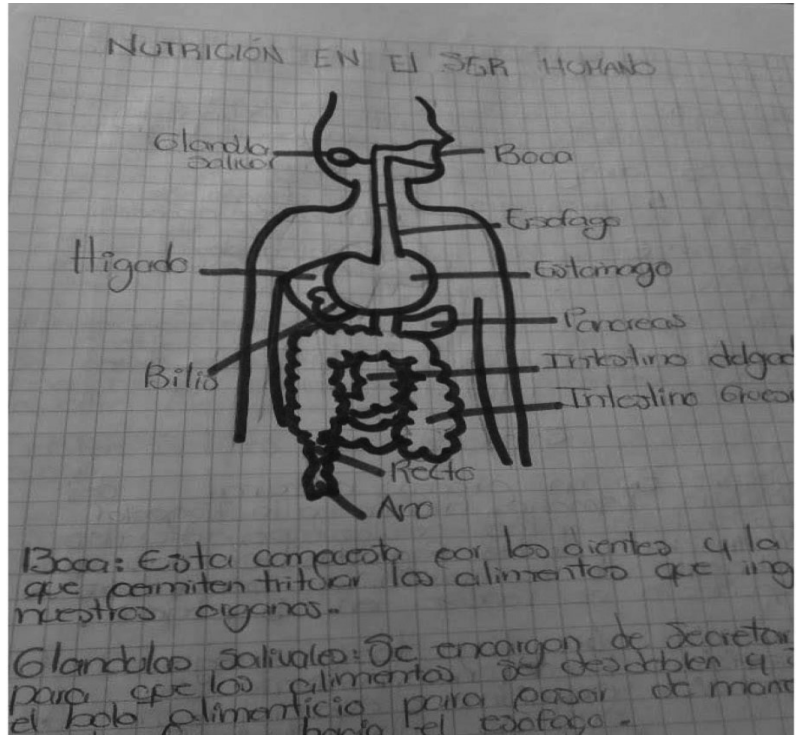

Figura 1. Nutrición en el ser humano

Fuente: elaboración propia.

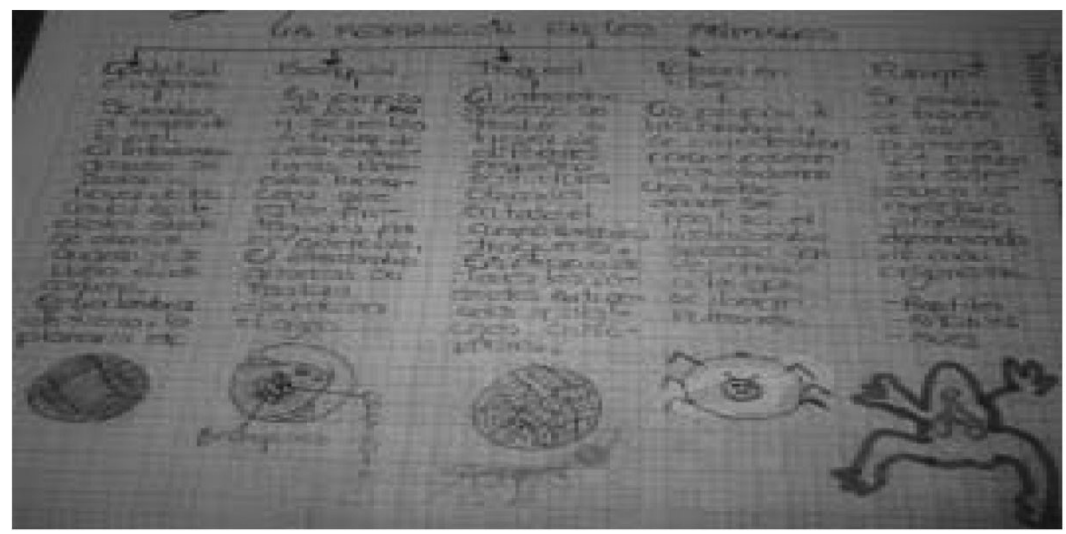

Figura 2. La respiración en los animales

Fuente: elaboración propia.

en clase. En algunos casos, la profesora dicta; en otros, explica, y ellos deben tomar nota. Se trata de que quede consignada la información con la representación gráfica del tema. A continuación se retoman dos explicaciones en donde se evidencia el valor que la docente le da a las representaciones gráficas:

\section{$A^{\circ}$ : Sístole y diástole.}

M: Silencio, bueno, ojo, aquí no va a ser fácil reconocer lo que es sístole y diástole porque, ¿qué es la sístole y diástole?
$A^{\circ}$ : Son laaa, los movimientos que tiene el corazón. M: Profesor: Movimiento del corazón. ¿Para qué servirán esos dos movimientos?

$A^{\circ}$ : Uno es de contracción y el otro es de (...).

M: Correcto, sístole es la contracción del corazón, o sea el corazón se contrae, es como cuando yo espicho una bolsa, si yo espicho una bolsa, ¿qué pasa con el líquido?

$A^{\circ}$ : Sale.

M: Cierto, una vejiga que yo espicho, que está llena de líquido, ¿sale?, sístole, ojo al movimiento, ¿cómo es?, lectura del movimiento sístole. Sístole, diástole, 
dos movimientos ¿Cierto? ¿Para qué sirven esos dos movimientos? ¿Para (...)?

$A^{\circ}$ : Para bombear.

M: Para bombear la sangre, ok, recibir y bombear sangre, y así sucesivamente.

La figura 3 fue la que empleó la profesora en sus diapositivas.

En otra ocasión la profesora explicó las redes y cadenas tróficas, y para ello realizó un dictado, en el cual iba explicando; además dibujó una pirámide alimenticia. La docente empleó las figuras 4 y 5 para ilustrar esos temas.

En los dos colegios observados se encontró que las profesoras algunas veces preparaban los temas a través de presentaciones en PowerPoint para mostrarles imágenes que aclararan y apoyaran las explicaciones verbales. La proyección de dibujos hizo que la comprensión de la clase se afianzara, dado que algunos estudiantes expresaron mayor entendimiento al observar imágenes que estimularan su proceso de aprendizaje. En concordancia con lo planteado por Barton y Hamilton (1998):

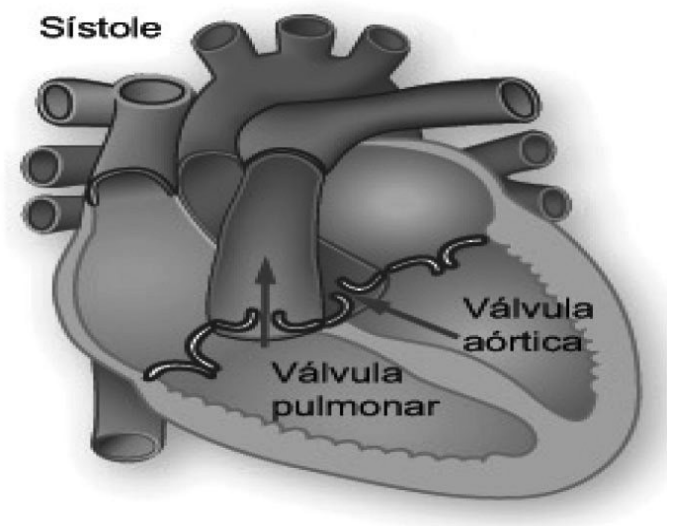

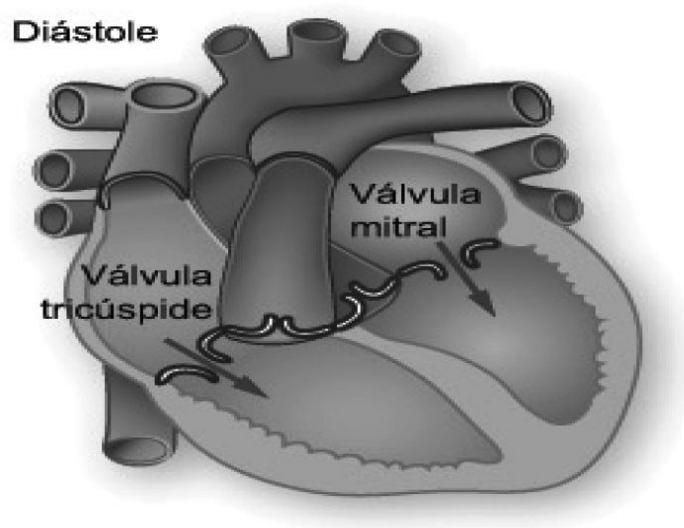

Figura 3. El latido cardiaco

Fuente: Texas Heart Institute (2015).

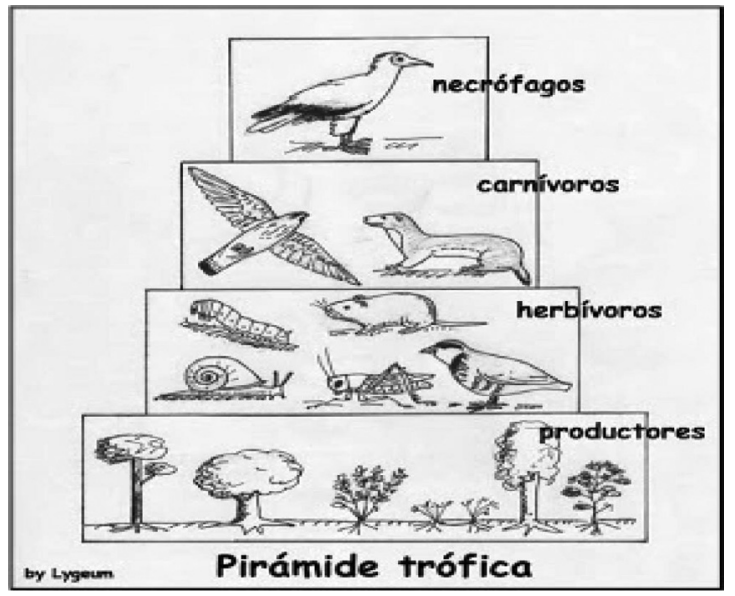

Figura 4. Pirámide trófica

Fuente: ¿Para qué futuro educamos? (2011). 


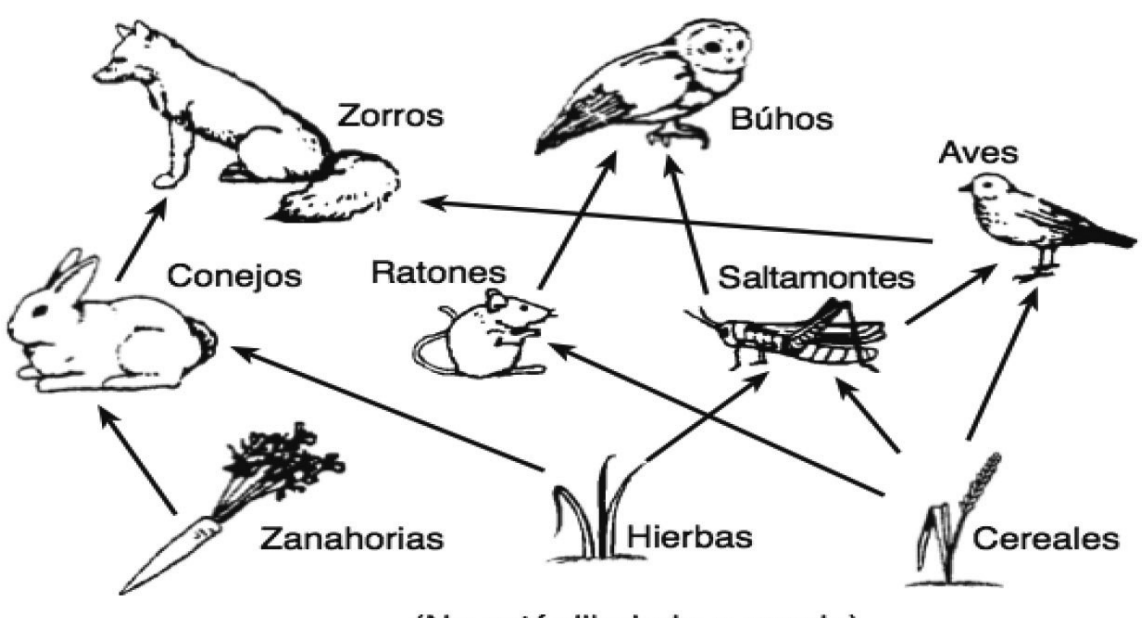

(No está dibujado a escala)

Figura 5. Dibujo para ejercitar sobre redes tróficas

Fuente: Ética y transformación del entorno (2015).

Las prácticas letradas implican la regulación social de los textos, es decir, quien tiene acceso a ellos y quien los puede producir. Los recursos a los cuales la gente tiene acceso pueden ser vistos en términos de destrezas técnicas y equipo, así como de sitios y apoyos para el aprendizaje que pueden tener base institucional o pueden ser informales (p. 126).

El uso del libro se hace evidente en una institución más que en la otra, sirve de pauta para desarrollar contenidos de manera ordenada y secuencial, es el apoyo para desarrollar tareas, resolución de talleres y guías propuestas por el docente. Estas acciones favorecen la relación del estudiante con el texto impreso.

Dentro de las prácticas de literacidad que se emplean en el aula de clase, se encuentra la lectura en voz alta, con el objetivo de que los estudiantes al escuchar a su compañero y seguir la lectura en el libro puedan comprender de una manera más efectiva las temáticas de la asignatura, pierdan el temor a ser escuchados, afiancen su personalidad y se estimule el debate participativo y crítico. El docente apela a estrategias que involucren a todos los estudiantes, como se ve en este segmento:

M: Entonces vamos a leer en voz alta, para que todos los escuchen.

\section{Evento letrado}

La segunda categoría corresponde a los eventos letrados, los cuales son visibles y se encuentran en las costumbres o prácticas cotidianas y están determinadas por el contacto entre los estudiantes y los profesores. Se trata de prácticas que estimulan la asimilación de hábitos o tradiciones personales en el contexto grupal y que son visibles a lo largo de la observación. Candela (1993) explica al respecto:

El conocimiento socialmente construido en el aula es, en ciertos momentos, un encuentro de argumentaciones como una forma de negociación y reconstrucción del conocimiento, mientras que se mantiene la comunicación. Las expresiones orales se toman entonces como significados construidos en un contexto de interacción, como productos de una confrontación en relación con otros y con una posición precisa en la conversación y no como evidencia de modelos cognitivos personales (p.33).

En la tabla 2 se presentan los eventos letrados que fueron más recurrentes en las dos instituciones, los cuales se definieron a partir de la observación.

A través de los textos los estudiantes reconocen y se aproximan a los temas. El abordaje de los contenidos en biología es una práctica epistémica de 
Tabla 2. Eventos letrados dominantes por institución

\begin{tabular}{|c|c|c|c|c|}
\hline $\begin{array}{l}\text { Eventos de } \\
\text { literacidad/ } \\
\text { instituciones }\end{array}$ & Eventos de literacidad & Contexto físico & Artefactos & Actividades visibles \\
\hline \multirow[t]{5}{*}{ Institución 1} & $\begin{array}{l}\text { Explicaciones a partir de } \\
\text { consultas de los estu- } \\
\text { diantes. }\end{array}$ & $\begin{array}{l}\text { El aula de clase, } \\
\text { organizada algunas } \\
\text { veces en mesa } \\
\text { redonda. En pocas } \\
\text { ocasiones en el } \\
\text { laboratorio de } \\
\text { ciencias. }\end{array}$ & $\begin{array}{l}\text { El cuaderno y el } \\
\text { libro de texto. }\end{array}$ & $\begin{array}{l}\text { La profesora genera preguntas y } \\
\text { discusión sobre los temas. A veces } \\
\text { solicita lectura del tema consulta- } \\
\text { do en enciclopedias, otras veces } \\
\text { se apoya en el libro de texto. }\end{array}$ \\
\hline & Dictado. & Aula de clase. & El cuaderno. & $\begin{array}{l}\text { La profesora dicta el texto y va } \\
\text { introduciendo preguntas, explica- } \\
\text { ciones, comentarios. }\end{array}$ \\
\hline & $\begin{array}{l}\text { Explicaciones a partir del } \\
\text { libro de texto, materiales } \\
\text { elaborados por la pro- } \\
\text { fesora, presentaciones } \\
\text { PowerPoint o proyección } \\
\text { de videos ilustrativos. }\end{array}$ & Aula de clase. & $\begin{array}{l}\text { El libro de texto y } \\
\text { el cuaderno. }\end{array}$ & $\begin{array}{l}\text { La clase se construye a partir de lo } \\
\text { consignado en el libro. }\end{array}$ \\
\hline & $\begin{array}{l}\text { Problematización de } \\
\text { los temas a partir de las } \\
\text { preguntas y las consultas } \\
\text { realizadas por los estu- } \\
\text { diantes y la relación de } \\
\text { los temas con la realidad } \\
\text { de estos. }\end{array}$ & Aula de clase. & El cuaderno. & $\begin{array}{l}\text { La formulación de preguntas y la } \\
\text { discusión prevalecen. }\end{array}$ \\
\hline & Exposiciones. & Aula de clase. & $\begin{array}{l}\text { Carteleras, cua- } \\
\text { derno. }\end{array}$ & $\begin{array}{l}\text { Los estudiantes se ponen en si- } \\
\text { tuación, se documentan sobre un } \\
\text { tema y explican. }\end{array}$ \\
\hline \multirow[t]{4}{*}{ Institución 2} & $\begin{array}{l}\text { Salidas de campo para } \\
\text { observar el mundo } \\
\text { natural. }\end{array}$ & Visita a granja. & El cuaderno & $\begin{array}{l}\text { La clase se construye a partir de } \\
\text { lo que observan en el entorno, } \\
\text { algunas veces la explicación se } \\
\text { apoya en una indagación previa } \\
\text { por parte del estudiante. }\end{array}$ \\
\hline & $\begin{array}{l}\text { Explicaciones a partir del } \\
\text { libro de texto, materia- } \\
\text { les elaborados por la } \\
\text { profesora, presentaciones } \\
\text { PowerPoint o proyección } \\
\text { de videos ilustrativos. }\end{array}$ & Aula de clase. & $\begin{array}{l}\text { El libro de texto y } \\
\text { el cuaderno. }\end{array}$ & $\begin{array}{l}\text { La clase se construye a partir de lo } \\
\text { consignado en el libro. }\end{array}$ \\
\hline & Dictado. & Aula de clase. & El cuaderno. & $\begin{array}{l}\text { La profesora dicta el texto y va } \\
\text { introduciendo preguntas, explica- } \\
\text { ciones, comentarios. }\end{array}$ \\
\hline & $\begin{array}{l}\text { Problematización de } \\
\text { los temas a partir de las } \\
\text { preguntas, las consultas } \\
\text { realizadas por los estu- } \\
\text { diantes y la relación de } \\
\text { los temas con la realidad } \\
\text { de estos. }\end{array}$ & Aula de clase. & El cuaderno & $\begin{array}{l}\text { La formulación de preguntas y la } \\
\text { discusión prevalecen. }\end{array}$ \\
\hline
\end{tabular}

Fuente: elaboración propia 
construcción de saberes, para lo cual se disponen en el aula diferentes estrategias y operaciones, como se describieron en la tabla 2. El trabajo de explicación y problematización de los temas se hace más evidente en unas clases; en otras, prevalece el dictado. En la observación fue importante analizar lo referido a la explicación, pues en los dos contextos se encontró la importancia de llevar a los estudiantes a hacer juicios y usar un lenguaje científico, como se muestra a continuación:

M: Ojo, pues, revisemos. Dos válvulas, ojo, una que se llama tricúspide, esta es la válvula tricúspide, esta es la válvula tricúspide; válvula bicúspide, son dos válvulas, válvula tricúspide y válvula bicúspide, son dos válvulas, ojo, si nosotros vamos ojo a recapitular las partes del corazón, recordemos cuál es cuál, cuál es la anatomía del corazón, el corazón tiene unas capas, son tres capas, cuáles son: tiene una capa externa, una capa media y una capa interna, tiene tres capas.

Algs. $A^{\circ}$ S: ¿La externa es para proteger el corazón?

M: Correcto, la externa es para proteger, la interna es para dar estructura $y$, pero, la media.

$A^{\circ}$ : ¿La exocardia, miocardio y mesocardio?

M: Correcto, son tres exocardio, miocardio, mesocardio, correcto muy bien. Pensemos en la válvula tricúspide ¿qué controla?

El metalenguaje empleado en ciencias se relaciona con construcciones epistémicas específicas, se trata de explicaciones, leyes, justificaciones (Penney, Norris, Phillips y Clark, 2003), de ahí la importancia de generar en clase discusiones, de posibilitar espacios para solicitar justificaciones y explicaciones. En estas aulas es muy frecuente el uso de las preguntas “¿Qué?", „¿Por qué?", „¿Para qué?", "¿Cómo?", las cuales son fundamentales en la construcción de argumentos en la clase.

\section{Comunidad de discurso}

El acceso a la comunidad de discurso se da a partir de las estrategias que emplea el docente; en ese sentido, su rol es indispensable, debido a que el dominio que este ejerce sobre la asignatura permite promover la participación o, todo lo contrario, limitar el aprendizaje del estudiante. Al observar las dos instituciones educativas, se identificó que si las clases se vuelven monótonas, el estudiante se dispersa y no se interesa por aprender y participar, sin embargo, en las clases observadas prevaleció la explicación y la ejemplificación de los contenidos, de modo que el estudiante se involucraba a pensar y reflexionar lo que vive en su contexto social. Aquí se presenta un ejemplo de interacción entre docentes y estudiantes.

M: qué quiere decir esto aquí, este sufijo bio.

Algs. $A^{o}$ s: bio, bio, vida.

M: De vida ¿cierto?, entonces, cuando yo digo factores bióticos ¿me estoy refiriendo a qué?

Algs. A $^{\circ}$ : a vida.

M: Pero vida ¿de quién?, ¿de los seres vivos? Entonces el ecosistema está conformado primeramente por los seres vivos, y por otro lado, por los factores a cuando yo veo que algo tiene una a- antes me está diciendo que no, que falta o sin.

" $A$ ०: Que no tiene vida".

"M: entonces está conformado por los seres muertos pero (...) Vamos a utilizar vocabulario (...) seres inertes.

El aula se considera una comunidad de discurso que procura compartir un lenguaje, la profesora en el caso anterior va buscando significados con los estudiantes. Cada cultura tiene su propio lenguaje, Lemke (1997, p.12) explica que este "lenguaje no es solo vocabulario y gramática: es un sistema de recursos para construir significados". A partir de la voz del estudiante y del profesor, se configuran subjetividades y se construyen saberes desde los conceptos previos que se tienen con respecto al tema o desde situaciones vividas, en otras palabras, también se apela al aprendizaje significativo que cada participante de esta comunidad pueda aportar en beneficio del grupo en general. Por ejemplo, en la regulación de las dinámicas del grupo se pueden encontrar elementos propios de cada comunidad en el caso que a continuación se 
cita sobre recomendaciones para controlar los nervios al hacer exposiciones:

M: Calmen los nervios ¿no? Uno a veces se come un dulce antes de (...) relajados o uno opta por coger un palito como Derly, mueve el palito, lo que hace es señalar, con eso está suprimiendo nervios jlisto! Técnicas para que ustedes trabajen sus exposiciones.

De otro lado, el trabajo en grupo o los talleres en pareja estimulan la apropiación del conocimiento a través de la interacción entre los estudiantes, además se refuerza el estudio de la terminología disciplinar, puesto que el contexto sociocultural y el lenguaje están supeditados a los espacios en donde se desarrollan. A través de la interacción se establecen patrones que conllevan a comprender las palabras o las relaciones entre los conceptos.

Dominio de la asignatura por parte del estudiante Esta categoría corresponde a la incursión y afianzamiento en las prácticas por parte de los estudiantes, que se dan de modo gradual y que están enmarcadas en el grado seleccionado. Se trata del nivel de familiaridad con las rutinas de discusión, explicación, con la terminología, con los modos en que busca acceder a la información. Se observó que en las dos instituciones hay un dominio básico de terminología que han empezado a interiorizar desde el grado sexto. Los docentes usan un lenguaje científico que no es desconocido por los estudiantes. Además se notó que el dominio de la clase es subjetivo, depende del desempeño de cada estudiante, si bien es cierto que un grupo de estudiantes puede mostrar poco interés por la asignatura, también se encuentran casos en los que hay preocupación por buscar e indagar, por ejemplo, como estrategias se encontró que algunos tratan de tomar nota de todo lo que el docente explica, otros estudiantes hacen uso del celular para buscar en internet lo que el profesor está señalando y otros usan los apuntes de los compañeros.
A través de las estrategias planteadas por las docentes se plantean oportunidades para problematizar y que los estudiantes aprendan ciencia como una práctica (Pickering, 1995, p. 3). Es decir que se integren cuatro dimensiones del trabajo disciplinar:

- Dimensión conceptual. ¿Cómo teorías, principios, leyes, ideas son utilizados por los actores para razonar?

- Dimensión social. ¿Cómo los actores están de acuerdo con las normas y las rutinas implicadas en el desarrollo, la crítica y el uso de las ideas?

- Dimensión epistémica. Se trata de la base filosófica en la que los actores deciden lo que conocen y por ello están convencidos de lo que saben.

- Dimensión material. ¿Cómo los actores crean, adaptan y usan herramientas, tecnologías y otros recursos para apoyar el trabajo intelectual de la práctica?

Estas dimensiones están implicadas en la práctica, en la medida que involucran la construcción del conocimiento científico y el razonamiento como partes de una red más amplia que incluye el discurso científico, unas normas de participación en situaciones de aprendizaje de ciencias, así como aspectos sociales, políticos y culturales del contexto (Bazerman, 1988; Longino, 1990).

Así mismo, las dimensiones resultan fundamentales para entender que el acceso y el dominio de las prácticas y de las disciplinas están insertos en una red que configura las dinámicas que se plantean en el aula de ciencias, que no necesariamente siempre son visibles y que dependen de la participación de los actores implicados. Por ejemplo, resultó muy ilustrador en los dos casos observados la preocupación de las profesoras por que los estudiantes usaran un lenguaje claro, conciso y científico que demuestra la preparación del tema: 
M: bueno, perdón, eh, tú (...) atentos. Atentos que el compañero va a participar con lo que le correspondió a él.

$A^{\circ}$ : Primero les voy a dar las primeras enfermedades y luego las voy a explicar una por una y les doy la definición, ah, y también (...). Bueno, el perfil lípido es muy importante que lo tengan en cuenta, porque cuando a uno se le pasa empezamos a tener enfermedades y si uno sobrepasa los 25 años empieza a contraer enfermedades circulatorias.

El dominio también se evidenció en las rutinas que pudieron ser observadas asociadas con la experimentación, la adquisición de conocimiento científico y la necesidad de comprobar las experiencias cotidianas con lo que se explica en el aula. Se encontraron experiencias en las cuales se animaba a los estudiantes a compartir y discutir sobre ideas científicas, más allá de repetir o reproducir afirmaciones o respuestas correctas. El dominio se evidenció en las pautas ofrecidas por las docentes para que definieran o resolvieran problemas científicos y los asociaran con su realidad. Se trató de un proceso de búsqueda, interrogación, indagación y discusión entre los participantes.

De otro lado, en la construcción de conocimiento científico y en el dominio, la utilización de herramientas tecnológicas además del pensamiento crítico estimula en los estudiantes la práctica de la lectura y la escritura, porque permiten analizar problemáticas desde la cotidianidad. Especialmente, esto se pudo encontrar en las presentaciones y en los videos que proyectaban las profesoras. Efectivamente el uso de las TIC, como lo señala Coll (2004) crea condiciones que pueden ser novedosas e interesantes para los estudiantes y que posibilitan ese dominio en los términos referidos anteriormente, dado que:

La novedad reside más bien en el hecho de que, a partir de la integración de los sistemas simbólicos clásicos (lengua oral, lengua escrita, lenguaje audiovisual, lenguaje gráfico, lenguaje numérico, etcétera), las TIC crean condiciones totalmente inéditas para operar con la información, representarla, procesarla, acceder a ella y transmitirla (p.12).
En ese sentido, a través del uso de las tecnologías se puede profundizar en los temas, la indagación de textos en cualquier formato (escrito, digital, audiovisual), generar mayor interés por la materia y fomentar prácticas de lectura y escritura asociadas con los intereses de los estudiantes y la asignatura.

\section{¿Qué mostraron las entrevistas realizadas a las docentes?}

A partir de lo indagado en las entrevistas, se pudieron identificar las siguientes categorías asociadas a las concepciones de las docentes, sobre la importancia de la lectura y la escritura en el desarrollo de su asignatura, así como de su uso: a) el uso de la lectura como recurso de investigación; b) el uso de la escritura como medio de producción desde conceptos interiorizados, y c) la enseñanza de la biología como una posibilidad de formar el pensamiento científico.

Con respecto al uso de la lectura como recurso de investigación, en las dos instituciones se promueve la indagación y lectura de textos para analizar temas científicos, se recurre a enciclopedias, al libro de texto de la asignatura, guías o escritos realizados por los docentes del área. Ante las dudas que surgían, los estudiantes buscaron la orientación del profesor, releyeron los textos varias veces, consultaron en el diccionario o en su celular el vocabulario desconocido. Sin embargo, los docentes de las instituciones manifestaron que uno de los obstáculos es el nivel de lectura. Al respecto, la profesora A expresó lo siguiente:

M: O sea, a veces no nos alcanza el tiempo para preparar la clase 90 minutos, son muy poco para que uno realmente logre interiorizar eso. Además, pues como que las necesidades de los estudiantes en este momento y de nuestros chicos no son esas, o sea ellos ven la lectura como una imposición como que lo tienen que hacer, o tienen que responder, mas no como que: "¡Oiga, ¿sí me va a servir para algo?!". Yo les digo a ellos: "Tómenlo no como un trabajo de clase, tómenlo para su vida eso les va a servir, eso les va a servir". 
En cuanto a la escritura como medio de producción desde conceptos interiorizados, algunos estudiantes presentan problemas para crear escritos propios, dado que se les dificulta expresar las ideas en un texto largo o con lenguaje desconocido, y cuando necesitan hacer estos escritos se apoyan en los apuntes que tienen en sus cuadernos. Por lo general, se encontraron definiciones, registros de tareas copiadas de internet o de enciclopedias, y prácticas de escritura asociadas al dictado, pero no producciones que evidenciaran una reflexión sobre los procesos o fenómenos naturales analizados. En la escritura se incluyen también herramientas, como los mapas conceptuales, cuadros sinópticos, informes o resúmenes, frente a los cuales muchas veces los estudiantes no tienen clara la idea de cómo se realizan o se estructuran.

El vocabulario que se exigía en las producciones escritas es el que se había manejado en la clase, de manera que a partir de ese trabajo el estudiante se familiarizó con una terminología especializada: además los escritos estuvieron sujetos a normas lingüísticas, gramaticales y ortográficas para que el mensaje fuera comprensible.

Cuando se indagó por la relación entre la asignatura y la formación del pensamiento científico, la profesora A, respondió lo siguiente:

M: Sí, para mí sí (hay relación), y obvio tiene que ser desde el enfoque del maestro, si digamos yo vengo y coloco aquí que la ley de Mendel es eso y lo doy por sentado que así es, el chico se va a quedar con eso; pero si yo le digo: "no, esto es una teoría y hay otras teorías", quizá, alguno, no todos se van a interesar por ir a buscar la otra teoría. Y para la siguiente clase me va a decir: "¡Profe, yo encontré que tal cosa también podría ser!". Entonces sí, a mí parece que desde la biología sí se puede incentivar ese pensamiento.

Frente a la misma pregunta la otra profesora respondió:

M: Sí, porque ellos hacen análisis a partir de lo que se sabe. Digamos estamos manejando un tema en específico "medio ambiente", entonces analizan y como que interiorizan también frente a esa problemática y se les empieza a ver sus trabajos. En este momento yo estoy trabajando el medio ambiente, y por ejemplo, me entregaron las producciones que yo quedo a veces sorprendida, porque estos chicos uno cree que los chicos no escriben, que no van a leer y consultar y resulta que sí leyeron y consultaron. Y como que se abrió la posibilidad, oiga los chicos sí están produciendo, pero producen es como cuando uno ha permitido esa interacción, como que no una imposición.

Las respuestas de las profesoras evidencian que ellas consideran que a través de sus clases pueden promover el pensamiento científico, lo cual implica generar situaciones de aprendizaje que propicien: la formulación de preguntas, el reconocimiento de la relación entre teorías para explicar los fenómenos naturales, la importancia de propiciar la generación de hipótesis y predicciones, la importancia de que los estudiantes indaguen en fuentes a las que tengan acceso, el reconocimiento del valor de la lectura y la escritura en la construcción de saberes científicos y el valor de la interacción y el diálogo entre ellos. En efecto, el papel del profesor resulta fundamental, puesto que puede configurar situaciones de trabajo didáctico y pedagógico que garanticen la participación productiva de los estudiantes y fomenten procesos de razonamientos a partir de los fenómenos naturales estudiados. A través del lenguaje, el profesor puede generar una comunicación activa, compartir ideas y andamios de aprendizaje entre los estudiantes (Berland y Hammer, 2012; Polman, 2004).

\section{CONCLUSIONES}

Las prácticas de literacidad en diferentes escenarios están determinadas por unas herramientas que pueden ser estructurales, humanas, artefactos materiales o tecnológicos, las cuales, a su vez, contribuyen en su configuración, para este estudio fue importante analizar las prácticas mediadas por textos en la clase de biología. Especialmente se pudo ver la actividad lectora ligada a los textos que se usan en ciencias, los cuales generalmente 
requieren de apoyos visuales. Leer en el escenario de la clase de biología se constituyó en una forma de acceder a la información, una actividad que cobró sentido gracias a las acciones de las profesoras, las estrategias y las tareas que les proponían a los estudiantes. Se reconoce que la dinámica y la interacción generada por ellas, se constituyen en un apoyo importante para potenciar un ambiente dialógico, en el cual prevalezca el interés por indagar y preguntar. Esto en concordancia con lo planteado por Candela (1999):

El discurso de los maestros y alumnos sobre la ciencia también incluye la organización social que se le da a ese discurso y los procedimientos por medio de los cuales se construye el conocimiento científico: el carácter impersonal que se atribuye a los hechos científicos, la necesidad de sustentarlos en "lo que se ve", la estructura argumentativa con la que se justifica una versión frente a otras alternativas explicativas, la búsqueda de coherencia (para los participantes) entre interpretaciones de fenómenos similares en diferentes marcos o contextos (papel de las metáforas y analogías) o la búsqueda de consen$\operatorname{sos}$ (p. 238).

El ingreso a una comunidad discursiva implica el dominio de habilidades comunicativas específicas, asumir roles y adquirir recursos lingüísticos como terminología especializada, en este caso resultó interesante ver cómo los estudiantes se aproximaban a temáticas que los llevaron a inquietarse por los órganos y los sistemas del cuerpo y su funcionamiento, por fenómenos naturales, la clasificación de los seres vivos y por enfermedades. Efectivamente, los estudiantes se acercan a la comprensión de los contenidos cuando interactúan en situaciones próximas a su realidad. Como lo plantea Sanmartí (2007) sobre los estudios que ha realizado con niños y niñas en primaria, y ha encontrado que "para explicar un fenómeno observado recurren constantemente a metáforas y analogías utilizando frases del tipo 'es como', 'es como si', 'es igual que', etc. Su capacidad de establecer relaciones es muy amplia en función de sus experiencias previas" (p. 3).
El dominio del lenguaje científico se logra, en parte, gracias al acompañamiento que el profesor hace a sus estudiantes sobre los conceptos o terminología desconocida. Se trata de un proceso progresivo en el cual estos se inician en la formación de su pensamiento científico desde sus propias experiencias, desde el estudio individual y grupal.

En los eventos letrados identificados en los contextos prevalece la exposición y la discusión de los temas, lo cual demanda una participación activa de los estudiantes, es decir que la voz de ellos se escuchó a través de actividades como las exposiciones, los resultados de búsquedas de información, la lectura en voz alta y las explicaciones espontáneas. Si bien se apoyan en prácticas de escritura, aún prevalece la transcripción y el dictado, no la reflexión sobre lo que escriben. En el caso de las instituciones analizadas para ampliar lo referido a las prácticas de escritura habría que poner en consideración las consignas de las profesoras, esto pensando en un futuro estudio.

En conclusión, la educación científica no debería enmarcarse en la memorización de patrones o en la repetición de procesos, los estudiantes deben ser parte de los procesos, deben estar preparados para que la ciencia apoye sus convicciones o inquietudes, no siempre definir que es ella quien tiene la última palabra. El dominio del lenguaje científico, tanto la producción escrita y la lectura se aprenden junto con el dominio de artefactos y mediaciones de diferente tipo (materiales, tecnológicos, experimentos, casos, problemas, etc.) que pueden acercar al estudiante a la problematización de fenómenos naturales. El acercamiento al estudio del aula puede brindar oportunidades para reconocer cómo se logran los dominios disciplinares, qué tipo de procesos "dialógicos de creación de conocimiento" (Duschl, 2008) se dan para involucrar a los estudiantes en prácticas epistémicas de enseñanza de la ciencia. 


\section{REFERENCIAS BIBLIOGRÁFICAS}

Barton, D. y Hamilton, M. (1998). Local Literacies. Reading and Writting in one Community. Londres: Routledge.

Barton, D. y Hamilton, M. (2000). Literacy practices. En: D. Barton, M.E. Hamilton y R. Ivanic Situated Literacies: Theorising Reading and Writing in Context (pp. 7-15). Londres: Routledge.

Bazerman, C. (1988). Shaping written knowledge: The genre and activity of the experimental article in science. Madison: University of Wisconsin Press.

Berland, L.K. y Hammer, D. (2012). Framing for scientific argumentation. Journal of Research in Science Teaching, 49(1), 68-94.

Candela, A. (1993). La construcción discursiva de la ciencia en el aula. Investigaciones en la escuela. México D.F.

Candela, A. (1999). Ciencia en el aula. Los alumnos entre la argumentación y el consenso. México: Paidós.

Candela, A. (2012). Un estudio etnográfico sobre la enseñanza de ciencias en las aulas de escuela primaria. En: A. Molina (comp.) Algunas aproximaciones a la investigación en educación en enseñanza de las ciencias naturales en América Latina (pp. 3962). Bogotá: Universidad Distrital Francisco José de Caldas.

Cassany, D. (2008). Una metodología para trabajar con los géneros discursivos de cada disciplina o licenciatura. Jornadas sobre Lenguajes de Especialidad y Terminología. Leioa: Instituto Universitario de Euskera, Universidad del País Vasco (UPV/EHU).

Coll, C. (2004). Psicología de la educación y prácticas educativas mediadas por las tecnologías de la información y la comunicación. Una mirada constructivista. Revista Electrónica Sinéctica, 25, 1-24. Recuperado de: http://www.redalyc.org/articulo. oa?id=99815899016

De Tezanos, A. (1998). Una etnografía de la etnografía. Bogotá: Antropos.

Duschl, R.A. y Osborne, J. (2002). Supporting and promoting argumentation discourse in science education. Studies in Science Education, 38, 39-72.
Duschl, R. (2008). Science education in three-part harmony: Balancing conceptual, epistemic, and social learning goals. Review of Research in Education, 32, 268- 291.

Ética y transformación del entorno (2015). Redes tróficas. Recuperado de: http://eticatranformacion. blogspot.com.co/2015_06_01_archive.html

Goetz, J.P. y Le Compte, M.D. (1984). Etnografía y diseño cualitativo en investigación educativa. Madrid: Morata.

Hamilton M. (2000). Expanding the New Literacy Studies: Using photographs to explore literacy as social practice. En: D. Barton, M. Hamilton y R. Ivanič (eds.). Situated Literacies: Reading and Writing in Context (pp. 16-34). Londres: Routledge.

Hammersley, M. y Atkinson, P. (1994). Etnografía. Métodos de investigación. Barcelona: Paidós.

Jiménez, M. y Sanmartí, N. (1997). ¿Qué ciencia enseñar: objetivos y contenidos en la educación secundaria? En L. del Carmen (coord.). La enseñanza y el aprendizaje de las ciencias de la naturaleza en la educación secundaria. Cuadernos de Formación del Profesorado, 9. Barcelona: ICE de la Universidad de Barcelona y Horsori Editorial.

Krippendorff, K. (1990). Metodología de análisis de contenido. Teoría y práctica. Barcelona: Paidós.

Lemke, J.L. (1997). Aprender a hablar ciencia. Barcelona: Paidós.

Longino, H.E. (1990). Science as social knowledge: Values and objectivity in scientific inquiry. Princeton, NJ: Princeton University Press.

López-Bonilla, G. (2013) Prácticas disciplinares. Prácticas escolares: Qué son las disciplinas académicas y cómo se relacionan con la educación formal en las ciencias y en las humanidades. Revista Mexicana de Investigación Educativa, 18(57), 383-412.

Márquez, C. y Prat, A. (2005). Leer en clase de ciencias. Enseñanza de las ciencias, 23(3), 431-440.

Moje, E.B.; Stockdill, D.; Kim, K. y Kim, H. (2011). The role of text in disciplinary learning. En: M. Kamil, P.D. Pearson, P. Mosenthal, P. Afflerbach y E.B. Moje (eds.). Handbook of Reading Research (Vol. IV, pp. 453-486). Mahwah, NJ: Erlbaum/Taylor \& Francis. 
Moje, E.B. (2007). Developing Socially Just Subject-Matter Instruction: A Review of the Literature on Disciplinary Literacy Teaching. Review of Research in Education, 31, 1-44.

Moje, E. (2008). Foregrounding the disciplines in secondary literacy teaching and learning: A call for change. Journal of Adolescent \& Adult Literacy, 52(2), 96-107.

¿Para qué futuro educamos? (2011). Ecosistema: pirámides, cadenas y redes tróficas. Recuperado de: http://paraquefuturoeducamos.blogspot.com. co/2011/10/ecosistema-piramides-cadenas-y-redes.html

Penney, K.; Norris, S.P.; Phillips, L.M. y Clark, G. (2003). The anatomy of junior high school science textbooks: An analysis of textual characteristics and a comparison to media reports of science. Canadian Journal of Science, Mathematics and Technology Education, 3, 415-436.

Pickering, A. (1995). The Mangle of Practice. Time, Agency, and Science. Chicago: The University of Chicago Press.
Polman, J.L. (2004). Dialogic activity structures for project-based learning environments. Cognition and Instruction, 22(4), 431-466.

Sanmartí, N. (1997). Para aprender ciencias hace falta aprender a hablar sobre las experiencias y sobre las ideas. Textos de didáctica de la lengua y de la literatura, 8, 27-40.

Sanmartí, N. (2007). Hablar, leer y escribir para aprender ciencia. En: P. Fernández (coord.). La competencia en comunicación lingüística en las áreas del currículo (pp. 103-127). Colección Aulas de Verano. Madrid: MEC.

Texas Heart Institute (2015). Heart Anatomy. Recuperado de: http://www.texasheart.org/HIC/ Anatomy_Esp/systole_sp.cfm

Zavala, V. (2008). La literacidad o lo que la gente hace con la lectura y la escritura. En: D. Cassany (ed.). Para ser letrados. Voces y miradas sobre la lectura (pp. 25-35). Barcelona: Paidós.

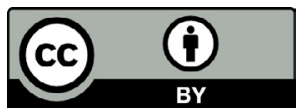

